ESJ Social Sciences

\title{
Consommation du «garba» en cote d'ivoire : entre risques sanitaires et construction de lien social
}

\author{
Aya Lydie Judicaelle Koffi, Doctorante
}

Unité de Formation et de Recherche des Sciences de l'homme et de la Société, Université Félix Houphouët-Boigny, Abidjan, Côte d'Ivoire. Centre Suisse de Recherches Scientifiques en Côte d'Ivoire, Abidjan, Côte d'Ivoire

Doi:10.19044/esj.2021.v17n19p230

Submitted: 05 February 2021

Accepted: 16 June 2021

Published: 30 June 2021
Copyright 2021 Author(s)

Under Creative Commons BY-NC-ND

4.0 OPEN ACCESS

Cite As:

Judicaelle Koffi A.L. (2021). Consommation du «garba» en cote d'ivoire : entre risques sanitaires et construction de lien social.

European Scientific Journal, ESJ, 17(19), 230. https://doi.org/10.19044/esj.2021.v17n19p230

\section{Résumé}

Selon les normes d'hygiène alimentaire en Côte d'Ivoire, les principales composantes (thon, attiéké, légumes frais et huile) destinées à la production du «garba» dépasseraient ces normes. Toutefois, cet aliment est consommé par la grande majorité de la population vivant à Abidjan et, le plus souvent en communauté. L'objectif de ce travail était de mettre en relief l'importance du «garba» dans la construction du lien social des consommateurs en dépit des risques sanitaires perçus. De ce fait, une étude quantitative et qualitative a été réalisée à Abidjan. Un questionnaire a été administré à 1067 consommateurs de « garba » en ce qui concerne l'approche quantitative et l'approche qualitative s'est basée sur trois études de cas. Le «triangle du manger» a servi de base théorique. Les résultats obtenus ont montré que les conditions de production et de vente du «garba» ont été perçues comme un facteur de fragilité physique chez $86,6 \%$ des consommateurs. Cependant, le «garba» est un support relationnel qui renforce la communion et la sociabilité des consommateurs grâce à son mode de consommation. En outre, des économies de 500 francs CFA sont réalisées par les consommateurs du «garba » sur chaque consommation, grâce à la valeur économique de l'aliment, à l'effet de consolider les liens sociaux dans leurs groupes d'appartenance. Le «garba» serait donc devenu un élément essentiel dans les habitudes alimentaires des citoyens abidjanais, puisqu'il permet d'entretenir les relations sociales et intervient dans la construction du lien social. 
Mots clés : Relation sociale, risques sanitaires, «garba», Abidjan, Côte d'Ivoire

\title{
Consumption of "Garba" in Cote d'Ivoire: Between Health Risks and Building of the Social Bond
}

\author{
Aya Lydie Judicaelle Koffi, Doctorante
}

Unité de Formation et de Recherche des Sciences de l'homme et de la Société, Université Félix Houphouët-Boigny, Abidjan, Côte d'Ivoire. Centre Suisse de Recherches Scientifiques en Côte d'Ivoire, Abidjan, Côte d'Ivoire

\begin{abstract}
According to food hygiene standards in Côte d'Ivoire, the main components (tuna, attiéké, raw vegetables and oil) to produce "garba" would be outside these standards. However, this food is consumed by most of the population living in Abidjan, most often in groups. The objective of this work was to highlight the importance of "garba" in building the social bond of consumers despite the perceived health risks. Therefore, a quantitative and qualitative study was carried out in Abidjan. A questionnaire was administered to 1067 consumers of "garba" regarding the quantitative approach and the qualitative approach was based on three case studies. The "eating triangle" served as a theoretical basis. The results obtained showed that the conditions of production and sale of "garba" were perceived as a factor of physical fragility by $86.6 \%$ of consumers. However, "garba" is a relational support which reinforces the communion and the sociability of the consumers thanks to its mode of consumption. In addition, savings of 500 CFA francs are made by consumers of "garba" on each consumption, thanks to the economic value of the food, to consolidate social ties in their home groups. "Garba" has therefore become an essential element in the eating habits of Abidjan citizens, since it helps to maintain social relations and intervenes in the construction of social links.
\end{abstract}

Keywords: Social relation, health risks, «garba», Abidjan, Côte d'Ivoire

\section{Introduction}

Le «garba» est un aliment de la restauration populaire, composé d'attiéké, de poisson thon frit, de légumes frais (piment, oignon, tomate), d'huile et de cube pour l'assaisonnement. Cet aliment est qualifié de mauvaise qualité sanitaire et nutritionnelle à cause des conditions de production et de vente qui ne respecteraient pas les normes d'hygiène alimentaire admises dans 
le pays (Koffi, 2013). En effet, les pratiques d'hygiène des vendeurs sont souvent désapprouvées par les consommateurs. Des griefs sont également faits sur la qualité et le type d'attiéké utilisé pour le "garba», qui est communément appelé "attiéké de type garba », mais aussi sur la qualité de l'huile de friture servant à la production de ce mets.

L'attiéké de type «garba» est généralement préparé en raccourcissant (ou en omettant) certaines étapes de production, entrânant ainsi des temps de fermentation et de cuisson inadéquats ( Heuberger, 2005; Djeni et al., 2011).

De plus, la qualité microbiologique de l'attiéké «garba» pourrait être à l'origine d'une toxi-infection (Bassa et al., 2016; Assanvo et al., 2019). Quant à l'huile des « garbadromes $»^{1}$, une étude en biochimie alimentaire réalisée par Diabaté et al. (2018) dans la ville d'Abidjan a montré que les pratiques de friture du poisson destiné à la préparation du «garba»par les acteurs de cette filière pourraient affecter la santé physique des consommateurs à cause de la réutilisation abusive de l'huile qui va jusqu'à sept fois. En plus de cela, les analyses microbiologiques réalisées par Anoman Koffi et al. (2018) et Anoman Koussemon et al. (2018) sur plusieurs échantillons de «garba »

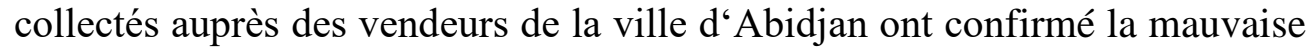
qualité microbiologique de cet aliment.

Malgré les risques sanitaires pouvant être associés à la consommation du «garba», l'attiéké de type «garba» est l'attiéké de grande consommation (Assanvo et al., Op.cit.) comme l'atteste la forte fréquentation des «garbadromes» à Abidjan. En effet, les «garbadromes»» sont les lieux de restauration les plus fréquentés par les ivoiriens (28\%) après les débits de boisson (44\%) (RTI, magazine Made in Africa, 2017). Un article de presse a révélé que le «garba» est l'aliment consommé par certains individus de la religion musulmane en début de jeûne pendant le mois de ramadan ${ }^{2}$. Aussi, il ressort une sorte de paradoxe entre le risque vital de consommation perçu au niveau du «garba» et les habitudes alimentaires des abidjanais. Ce paradoxe entre les pratiques alimentaires des abidjanais et leurs discours sur la qualité du «garba» confirme les propos de Claudian et Trémolières (1978), cités par Poulain (2002, p. 200) qui indiquent que: "L'homme de l'an 2000 se trouve dans une curieuse alternative. Il connaît de mieux en mieux les dangers des excès ou des carences relatives à son alimentation. La science n'est pas loin de pouvoir dire ce qu'il faut faire pour être en bonne santé et donc pour qu'il n'y ait plus qu'à se conformer à ses prescriptions. Mais derrière le manger, il y a ce que devient l'homme qui mange, qui lui importe encore plus ". Quant à Corbeau et Poulain (2002), ils ont pu appréhender cette contradiction en

\footnotetext{
${ }^{1}$ Lieux de production, de vente et/ou de consommation du «garba»

${ }^{2} \mathrm{http}: / /$ raphaeltanoh.blogspot.com/2010/10/ces-garbadromes-de-4-heures-du-matin.html, consulté le 18 Octobre 2020.
} 
montrant que le consommateur est libre dans certaines sphères, mais conditionné dans d'autres. Ils concluent par conséquent que le consommateur est «pluriel».

Le caractère «pluriel» des consommateurs a été observé chez des abidjanais. Il s'agit des individus qui ont intégré la consommation du « garba » dans leurs habitudes alimentaires, bien qu'ils l'eussent perçu comme un aliment de mauvaise qualité sanitaire et nutritionnelle. Deux périodes ont pu marquer la vie de l'un des individus dans son rapport avec le «garba »: une période de faible consommation et une période de grande consommation. $\mathrm{La}$ période de grande consommation est justifiée par la valeur sociale que l'individu associe à l'aliment. Les deux autres individus n'ont pas connu de période de faible consommation, mais consomment le «garba» bien qu'ils le perçoivent comme un aliment inférieur, mais auquel ils attribuent une valeur sociale.

La valeur sociale de l'alimentation a été prouvée par Corbeau (2010) à travers la théorie dénommée «triangle du manger». Selon cet auteur, cette théorie permet d'expliquer la dimension plurielle des consommateurs en envisageant un consommateur identifié consommant un aliment identifié dans une situation particulière. Ayant appliqué cette théorie à l'explication du décalage entre la représentation des hamburger restaurants dans les discours dominants et les pratiques alimentaires, l'auteur a montré que le fait alimentaire qu'il appelle la «filière du manger» n'est pas réductible à sa dimension nutritionnelle, ni à sa dimension symbolique. Selon l'auteur, l'alimentation intervient dans la socialisation, dans les phénomènes d'appropriation culturelle. Ainsi, l'auteur montre que sa médicalisation, quoiqu'excessive ne détériore pas l'intérêt qu'elle joue dans la construction de l'équilibre affectif du consommateur et du lien social.

Paugam (2008) appréhende le lien social comme la relation entre l'individu et les membres de son réseau social «et des conditions du changement social de longue durée ». Selon l'auteur, dès sa naissance, l'individu se trouve dans une relation d'interdépendance avec les autres et la solidarité est le socle de ce qu'il appelle l'homo-sociologicus à tous les stades de socialisation. Inscrit dans la sociologie de l'alimentation, cet article vise à déterminer l'importance du «garba » dans la construction du lien social en dépit des risques sanitaires associés à sa consommation. Pour se faire, il s'agira de déterminer la perception des conditions hygiéniques de production et de commercialisation chez les consommateurs, ensuite d'appréhender les valeurs sociales du « garba ». Pour la détermination des valeurs sociales du « garba », l'accent sera mis sur le lien de filiation, c'est-à-dire lien entre parents et enfants, et le lien de participation élective qui est le lien entre conjoints, amis, proches choisis, définit par Paugam (Op.cit.). 


\section{Méthodologie \\ Zone d'étude}

Ce travail a été mené en 2018 et 2019 auprès des consommateurs de «garba » (Figure 1) de la ville d'Abidjan, située au sud-ouest de la Côte d'Ivoire, et plus précisément dans les communes de Yopougon, Port-Bouët et Abobo (Figure 2).

Le choix de la ville d'Abidjan s'explique par le fait qu'Abidjan est le lieu de grande production et de consommation du «garba » dans le pays (Abouya, 2011). Quant aux communes d'étude, elles ont été tirées de façon aléatoire car la production et la consommation du « garba » se font de la même manière dans toutes les communes d'Abidjan. Les noms des communes ont donc été inscrits sur des bouts de papiers et mis dans une boite, puis un tirage sans remise a été fait.
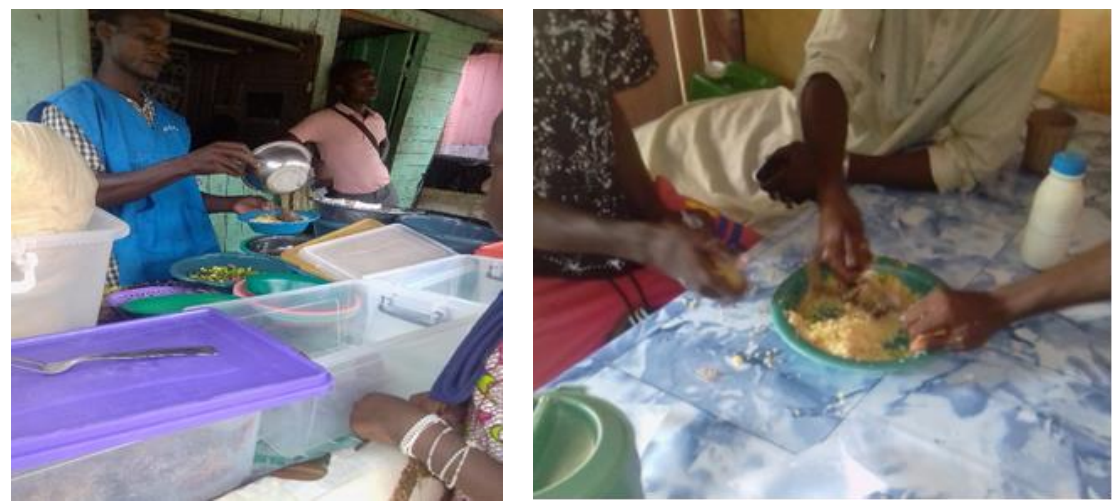

Figure 1 : a : un garbadrome situé dans la commune de Yopougon; b : vue des individus consommant le «garba » dans un « garbadrome».

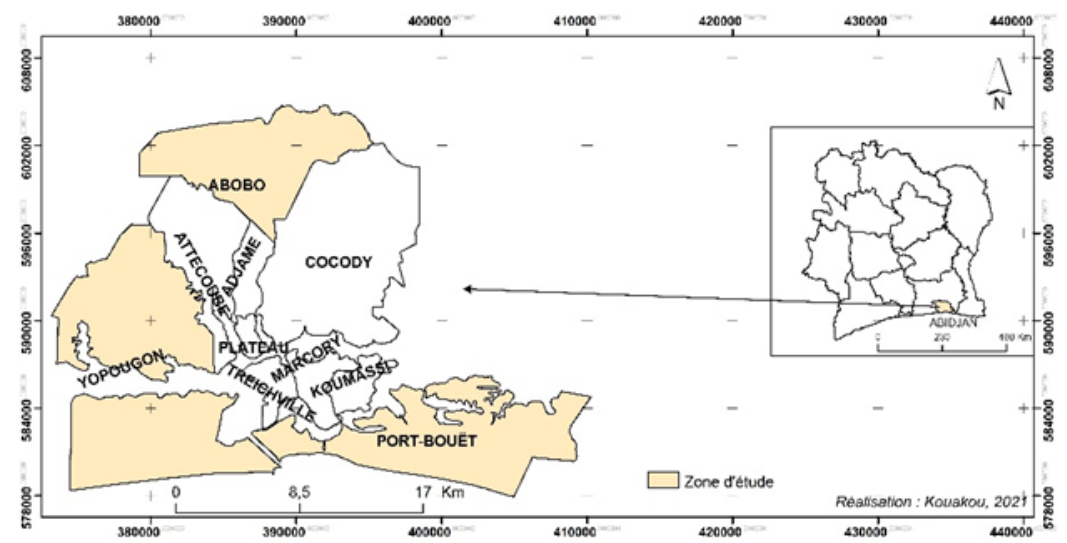

Figure 2: Carte de la zone d'étude montrant les communes d'étude Source : BENETD (2018). 


\section{Matériel et méthodes}

Cette étude sociologique à la fois quantitative et qualitative a été faite à partir des entretiens par questionnaire et des interviews semi-structurées. Au total 1067 individus ont été interrogés à partir des questionnaires en utilisant la technique d'échantillon accidentel (N'Da, 2006). L'objectif de cette enquête par questionnaire était de déterminer la perception de la qualité sanitaire et nutritionnelle du «garba » chez les consommateurs de «garba». La taille de l'échantillon a été déterminée à partir de la formule 2 de l'OMS (Vaughan et al., 1991).

$$
\mathrm{n}_{0}=\frac{\mathrm{pq}}{(\mathrm{E} / 1,96)^{2}}
$$

Où $\boldsymbol{n}_{0}$ est la taille de l'échantillon pour une population-mère indéfinie, $\boldsymbol{p}$ représente le taux de consommation de «garba» à Abidjan, $\mathrm{p}=50 \%$ lorsque la taille de la population n'est pas connue (Vaughan et al., 1991). $\boldsymbol{q}=100-\mathrm{p}$, $\boldsymbol{E}$ est la marge d'erreur tolérée (\%) estimé ici à $3 \%$.

Des études de cas à travers des entretiens semi-structurés avec trois consommateurs majeurs, sélectionnés de façon aléatoire dans les " garbadromes » ont été également réalisées. Ces consommateurs étaient tous issus de la classe populaire avec des âges compris entre 25 ans et 30 ans. Au niveau théorique, le «triangle du manger» de corbeau (2010) s'est révélé intéressant pour la compréhension de ces cas. Selon l'auteur, parler d'alimentation met en exergue un consommateur identifié, un aliment identifié et une situation particulière justifiant la consommation de l'aliment. C'est ce qui permet d'avoir les trois sommets d'un «triangle du manger» en interaction perpétuelle. Pour des raisons pédagogiques, l'auteur décrit les caractéristiques d'identification des trois composantes du triangle.

Selon Corbeau, le consommateur a une dimension diachronique et synchronique dans ses mutations. L'auteur décrit le consommateur comme étant un individu aussi bien producteur que reproducteur de modèles alimentaires, de principes de comportements alimentaires. Il a des trajectoires socioculturels pluriels. De ce fait, ses choix alimentaires, son mode de consommation et autres varient en fonction de sa position sociale (Bourdieu, 1979; Lambert, 1987). Par ailleurs, le sexe et l'âge déterminent aussi la consommation alimentaire de l'individu (Fischler, 1990; Garabuau, 2002). Le mangeable et le non mangeable sont également des notions dépendant de la région d'origine de l'individu (Poulain, 1998), de ses croyances religieuses et éthiques.

$\mathrm{Au}$ niveau de l'aliment, Corbeau affirme qu'il doit être aussi appréhendé de façon plurielle. Leur variation dans le temps est liée à la technique de production. Il varie aussi dans l'espace à travers le goût, le prix 
et l'offre. Par leur histoire et par leur côté ordinaire, festif, exceptionnel, les aliments véhiculent des symboliques. Les consommateurs les perçoivent, plus ou moins inconsciemment, comme correspondantes à leurs attentes, comme provocatrices de peurs ou les rassurant (Corbeau, Op.cit., p 13).

Concernant la situation, l'auteur révèle qu'elle est le contexte social ou l'interaction entre le consommateur et l'aliment.

L'aliment concerné dans l'étude est le «garba». Selon Abouya (Op.cit.), le terme «garba» est né dans les années 1980. En effet, «Garba» était le nom de l'assistant de l'initiateur du mets. Ce terme a désigné le lieu de restauration du mets avant d'être assimilé à l'aliment. La composition du plat se fait en fonction des préférences des consommateurs. Ainsi, il y a des plats composés d'attiéké et de poisson thon frit uniquement (attiéké, poisson thon frit et piment), attiéké, poisson thon frit, piment, oignon, etc.

Au niveau des consommateurs de «garba», en 1980, on rencontrait deux (2) types de consommateurs de «garba». Une minorité de nationaux le considérait comme un en-cas (Abouya, Op.cit.). La grande majorité des consommateurs de «garba» était les individus de nationalité nigérienne dont la plupart était des employés du port autonome d'Abidjan. Dans les années 1990, à la faveur de la crise économique, une mutation de la représentation du «garba» s'est opérée chez les nationaux, surtout dans la gent estudiantine. Les étudiants ont donc adhéré massivement à la consommation de cet aliment. Aujourd'hui, le «garba» est consommé par la plupart des catégories socioprofessionnelles, mais surtout par les individus des quartiers populaires; les jeunes et les enfants (Koffi, Op.cit.).

L'enquête par questionnaire a été réalisée avec le logiciel ODK Collect version 1.30.1. Les tablettes ont donc été les supports physiques de collecte des données de terrain. Après la phase de collecte, les données ont été exportées sur le logiciel SPSS version 24 pour les analyses. Il s'agissait de déterminer le rapport que les consommateurs font entre la santé et les conditions de production et de vente du « garba».

Les entretiens semi-structurés ont été transcrits manuellement sur l'éditeur Word et analysés sur le logiciel NVivo version 12. L'analyse de contenu thématique développée par Bardin (2007) a permis d'appréhender les différentes valeurs sociales du «garba » : le «garba » perçu comme support de convivialité, le «garba » perçu comme facteur de construction de lien social dans les relations familiales et le «garba » perçu comme facteur de construction de lien social entre conjoints. 


\section{Résultats}

Conditions d'hygiène lors de la production et la commercialisation du « garba " perçues comme facteur de transmission de maladies chez les consommateurs

Un total de 1067 consommateurs de " garba » a été interrogé à l'aide d'un questionnaire dans le cadre de cette étude pour appréhender leur perception du lien entre les conditions d'hygiène lors de la production et la commercialisation du «garba » et la santé. Parmi eux, 86,6\% (n=924) ont répondu que les conditions de production et de vente du «garba» sont susceptible de rendre malade (figure 3).

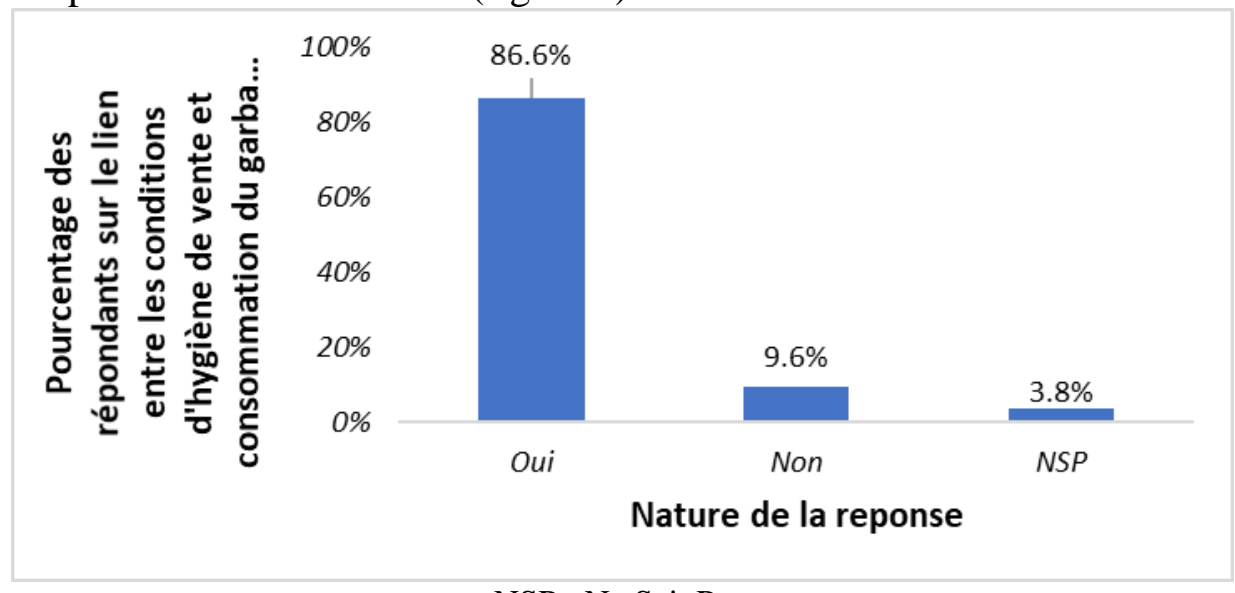

NSP : Ne Sait Pas.

Figure 3 : Perception des consommateurs par rapport au lien entre les conditions d'hygiène lors de la production et la vente du «garba » et la santé

Seuls 9,6\% ( $\mathrm{n}=102)$ des répondants ont affirmé le contraire tandis que $3,8 \%(\mathrm{n}=41)$ n'ont pas pu se prononcer sur la question.

Tous les répondants consomment l'aliment bien que la grande majorité ait affirmé que les conditions de production sont susceptibles de rendre malade. L'impact direct du «garba»sur la santé des répondants a donc été appréhendé.

Il est ressorti, selon les propos des enqupêtés que plus de la moitié d'entre eux, c'est-à-dire $73,3 \%(n-=924)$ de ceux qui ont affirmé que le «garba» pourrait rendre malade n'ont jamais été malades après consommation de l'aliment. Seulement le quart de ceux-ci, c'est-à-dire 25,3\% $(n=234)$ des répondants ont contracté une maladie après consommation du «garba». Enfin, $1,4 \%(\mathrm{n}=149)$ des répondants ne pensent pas avoir contracté une maladie après consommation du «garba».

Ces données quantitatives ont été justifiées par des entretiens semistructurés. En effet, selon les propos d'un répondant, sa perception de la qualité sanitaire et nutritionnelle a été influencée par les conseils nutritionnels 
de ses parents. L'individu a affirmé que selon ses parents, les éléments composants le « garba », notamment l'huile et l'attiéké, ne sont pas bons pour la santé. L'hygiène des acteurs de cette filière a été également remise en question par ses parents. Cette représentation de la qualité sanitaire et nutritionnelle du «garba » l'a conduit à un rejet de l'aliment jusqu'à une période récente (un an avant l'enquête).

"Moi-même, avant, je n'aimais pas trop le garba à cause de l'huile. Mes parents me disaient que l'huile du garba n'est pas trop bonne pour la santé. Et puis, ils me disaient aussi que les vendeurs n'étaient pas trop propres". (Entretien semi-structuré avec un étudiant, 25 ans, Yopougon, Avril 2019)

La perception de la qualité hygiénique et nutritionnelle que les parents ont du «garba» a impacté négativement sur les habitudes alimentaires du consommateur. Cela a amené l'individu à modifier la composition de son plat. Le consommateur faisait le choix de consommer uniquement le poisson thon des «garbadromes », car l'huile et l'attiéké de ces espaces gastronomiques étaient perçus comme impropres à la consommation. De plus, les autres répondants perçoivent l'huile des «garbadromes » comme impropre à la consommation. "Leur huile là même, souvent c'est noir comme ça". (Entretien semi-structuré avec un commerçant, 28 ans, Yopougon, Avril 2019).

Bien que les individus perçoivent le «garba » comme un aliment de mauvaise qualité sanitaire et nutritionnelle, ils le consomment pour diverses raisons, en l'occurrence pour des avantages sociaux.

\section{«Garba», support de convivialité}

Dans l'espace académique et ludique des répondants, la consommation du «garba» se fait souvent en groupe et avec la main. Ce mode de consommation du «garba» produit chez les individus un support relationnel. Il s'agit ici de la convivialité qui participe à renforcer les liens entre les commensaux.

"A l'école, on mange en groupe, ça renforce encore les liens d'amitié. Ça fait que souvent, quand on finit de manger, on peut s'asseoir et dire «ah, toi là, tu prends de gros coups êh, toi là, c'est comme ça que tu manges! " ça envoie un peu les causeries et puis on rit. On se moque un peu de l'autre. C'est intéressant quoi. Souvent, on peut balancer sur facebook, commenter". (Entretien semi-structuré avec un étudiant, 25 ans, Yopougon, Avril 2019).

Le mode de consommation du «garba» amène les individus à se passer des manières de table dans des groupes d'amis inscrits dans l'espace académique des enquêtés. Ils le mangent avec gourmandise, ce qui participe à 
l'ambiance autour du repas. L'individu qui accepte de manger le «garba» en groupe dans cet espace social doit donc être ouvert à tout sujet de plaisanterie relatif à sa manière de table. Les convives acceptent les moqueries de tout genre, quel que soit le moyen de communication utilisé pour diffuser l'information. Pour les amateurs du «garba» de ces groupes sociaux, la consommation de ce mets fait partie des moments de communion au sein du groupe car toute l'ambiance autour de l'aliment participe à renforcer les liens d'amitié.

Au-delà de l'espace académique, l'espace sportif des répondants est également un lieu de communion autour du «garba».

"Les soirs quand on a fini de jouer au ballon ou on a fini de s'amuser avec les amis, c'est toujours bon d'aller s'asseoir autour d'un plat de "garba» parce que c'est un truc qui se mange rapidement et puis du fait que ça se mange avec la main, je pense que c'est un facteur qui réunit les gens". (Entretien semi-structuré un agent marketeur, 30 ans, Yopougon, avril 2019).

Ces propos des personnes interviewées revèlent que le centre d'intérêt commun (passion, loisir, profession, etc.) est un facteur qui réuni les individus autour d'un plat de «garba». En effet, le sport et les loisirs étant des occasions de communion, les amateurs du «garba» matérialisent aussi cette communion par le partage du «garba» en commun. Les commensaux ne s'attardent pas sur les formes particulières des manières de table, d'où le fait de manger rapidement et avec la main. Cette simplicité dans la manière de consommer l'aliment pourrait briser les barrières sociales et participer ainsi à la communion entre les individus dans ces groupes sociaux.

\section{«Garba», facteur de construction de lien social dans les relations familiales}

Le «garba» serait consommé par les personnes enquêtées pour consolider leurs rapports dans l'espace familial. En effet, les résultats de $1^{\prime}$ enquête par questionnaire ont montré que le caractère bon marché du «garba » est la raison la plus évoquée par les répondants pour justifier leur attachement à l'aliment (figure 4). 


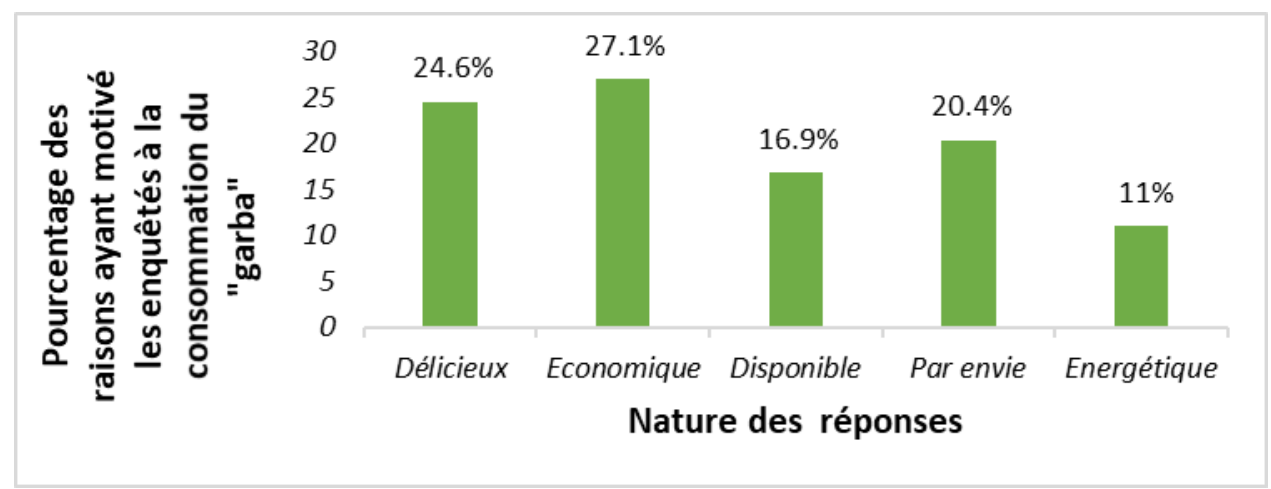

Figure 4: Raisons ayant motivé les enquêtés à consommer le "garba"

Le caractère bon marché du « garba » a représenté $27,1 \%$ des réponses. cette modalité de réponse est suivie de trois autres: le goût délicieux $(24,6 \%)$, l'envie $(20,4 \%)$ et la disponibilité de l'aliment (16,9\%). L'effet rassasiant du «garba » a représenté $11 \%$ des raisons ayant motivé la consommation de l'aliment chez les individus à l'étude. Le caractère bon marché et l'effet rassasiant de l'aliment permettent aux répondants de construire une rationalité économique qui les positionne dans des formes d'entraide participant à la construction d'un statut social valorisant dans leurs relations familiales. Au fait, dans les rapports entre consanguins, un répondant qui occupe une position d'ainé est animé par la « volonté de puissance » non apparent vis-à-vis de ses cadets. Ainsi, le choix stratégique du «garba» perçu comme bon marché et rassasiant lui permet de réaliser des économies pour apporter des aides ponctuelles aux cadets. Les économies réalisées à partir de la consommation du «garba» repose quelque fois sur des renoncements à ses envies personnelles. Cependant, l'investissement de ces économies dans les relations favorise le respect, la considération et la confiance des cadets vis-à-vis du répondant.

"Quand je vais à l'école, si j'achète du pain le matin, à midi encore, je suis obligé d'acheter attiéké pour tenir. Je vais me retrouver à 1000 francs de dépenses. . Donc, je préfère manger le "garba», comme ça, au lieu de dépenser 1000 francs, je dépense $500 \mathrm{~F}$ et puis je garde $500 \mathrm{~F}$ à côté.. Ça me permet de faire des transferts d'argent à mes petites sœurs. Tous ces gestes apportent le respect surtout parce quand tu es le grand frère, quand elles te demandent et puis tu donnes, même si ce n'est pas beaucoup, quand elles viennent, tu remarques qu'il y a toujours le respect. Voilà! Or, quand il n'y a pas ça là, c'est vrai, le respect est là, mais tu n'es pas trop considérée. (Entretien semi-structuré avec un étudiant, 25 ans, Yopougon, Avril 2019).

Le caractère bon marché et l'effet rassasiant du « garba » interviennent aussi dans la consolidation des rapports entre un répondant et ses géniteurs. Il 
permet au répondant financièrement indépendant, mais issus de condition économique modeste de réaliser des économies.

"Le garba me fait économiser plus par rapport au riz. Si c'est le riz que je mange, dans le mois ce que je peux dépenser là, ça sera très beaucoup parce que le riz, si je mange pour 1000 francs à midi, le soir 1000 francs, ça fait 2000 francs. Dans le mois, je vais me retrouver à 60000 francs de dépenses pour manger seulement. Or, si c'est attiéké, ça fait 500 francs de dépenses. Si j'ai mangé 500 francs à midi, le soir j'ai mangé 500 francs, ça fait 1000 francs. Dans le mois çà fait 30000 francs. Comme moi je ne mange pas attiéké la nuit, c'est la journée seulement, ce n'est pas tous les jours aussi que je le mange. Donc dans le mois, attiéké, je peux manger pour 13000 francs comme ça. En tout, je peux dépenser 40000 francs comme ça dans la nourriture dans le mois ". (Entretien semi-structuré avec un commerçant, 28 ans, Yopougon, Avril 2019).

Les économies réalisées par le répondant grâce au choix du «garba» dans ses habitudes alimentaires sont utilisées pour soutenir financièrement ses parents. Un canal de bénédiction s'établit alors entre l'individus et ses géniteurs. En effet, cet individu établit des relations distantes avec ses géniteurs grâce au gage symbolique (l'argent) avec lequel il les soutient. Sa présence auprès de ses parents se matérialise symboliquement par le soutien financier qu'il leur apporte, bien qu'étant physiquement loin d'eux. Il participe ainsi au bien-être de ses géniteurs. Selon le répondant, ce soutien financier lui permet de gagner la considération et la bienveillance de ses parents. Cela se matérialise par des appels téléphoniques et des paroles de bénédictions. Ce soutien financier brise la distance géographique et réorganise alors les rapports avec ces derniers.

\section{«Garba», facteur de construction de lien social entre conjoints}

La valeur économique et l'effet rassasiant du «garba » sont aussi mobilisées par les individus pour consolider leurs rapports avec leurs conjointes. En effet, un répondant issu de condition économique modeste convertit socialement les économies réalisées grâce à la consommation du «garba» auprès de son amie intime.

"Si j'ai entrepris une activité qui m'a permis d'avoir de l'argent, je vais dépenser, mais grâce à la consommation du garba, l'argent va tenir un peu longtemps sur moi. Donc, si souvent ma chérie me demande de lui faire des transferts d'unités de $200 \mathrm{~F}$ ou bien les petits trucs comme ça, grâce aux petites réserves que je fais, j'arrive à faire ça. Tout ça aussi amplifie notre relation, parce qu'elle a vu que je suis un gars, même si je n'ai pas beaucoup d'argent, mais au moins il y a quelque chose qui est sur moi, donc à tout moment si elle demande le minimum, elle peut trouver. Donc ça fait qu'elle se 
confie beaucoup à moi“. (Entretien semi-structuré avec un agent marketeur, 30 ans, Yopougon, Avril 2019).

Une volonté de valorisation de soi ou de prestige social ressort à travers les propos de ce répondant. Des stratégies sont alors adoptées pour gérer le revenu irrégulier et maigre du ménage sur une longue période. La gestion des ressources financières permet de satisfaire les besoins les plus élémentaires et les moins onéreux de sa compagne. Ainsi, un climat de confiance s'établit et favorise les confidences.

\section{Discussion}

Au-delà de la qualité hygiénique et nutritionnelle perçue comme inférieure, le «garba » consolide les rapports chez les répondants dans leur milieu familial et extra-familial. Cet côté relationnel de l'aliment encourage les répondants à le consommer, montrant ainsi le caractère pluriel et complexe du comportement alimentaire (Corbeau, 2010; Laporte et al., 2015). En effet, la consommation du «garba » est appréhendée comme un facteur d'inclusion dans des groupes d'amis de jeunes. Par conséquent, l'individu qui ne consommait pas cet aliment du fait des conditions de production et de vente non hygiéniques a fini par l'intégrer dans ses habitudes alimentaires à cause de son réseau d'amis. La situation de convivialité dans laquelle se trouve l'individu modifie donc sa perception du «garba ». Ce résultat corrobore le « triangle du manger » de Corbeau qui a montré que la situation du mangeur modifie ses attentes. Elle peut aussi valoriser certains aspects de l'aliment et développer la sociabilité entre les consommateurs (Corbeau, Op.cit.).

Dans ces réseaux d'amis, la consommation du «garba» devient comme un facteur d'identité sociale car des sujets de plaisanterie visant à renforcer les relations d'amitié se font autour de l'aliment. D'ailleurs, l'alimentation en tant que facteur d'identité sociale a été aussi appréhendée par Tibère (2006) au sein de la communauté réunionnaise. Cet auteur a montré que la pratique du modèle alimentaire régi par la communauté est nécessaire pour être reconnu comme membre de la société réunionnaise. L'alimentation devient ainsi un facteur d'exclusion ou d'intégration, selon que l'individu l'accepte ou la rejette. Au-delà de l'adhésion au modèle alimentaire, d'autres auteurs ont montré que le partage du repas en commun construit les relations sociales. En effet, Hastorf (2016) a affirmé que dans certaines sociétés, manger seul est considéré comme inapproprié, même sinistre. A cet effet, Dunbar (2017) a montré qu'au Royaume-Unis, les personnes qui mangent avec les autres se sentent plus en bonne santé et ont un réseau social plus vaste qui leur fournit un soutien social et émotionnel. Il affirme que les rires, les causeries de tout genre rapprochent les commensaux et permettent un mieux être. Selon Rezende (2007), cette quête de mieux-être à travers l'alimentation amène les travailleurs anglais de la classe bourgeoise à investir le temps et l'énergie qui 
sont des facteurs précieux dans le domaine du travail pour consolider leur rapport avec leurs amis autour d'un repas.

La satisfaction du besoin relationnel est également recherchée dans les relations familiales à travers la consommation du «garba ». En effet, la rationalité économique autour du «garba » permet aux individus de la classe populaire de protéger leur statut social dans la sphère familiale. Ils mobilisent donc des ressources économiques grâce à la consommation du «garba » en vue de conquérir leurs proches. Le maintien des rapports sociaux avec les proches à travers la mobilisation du capital économique a été appréhendé par Giddens, cité par Delannoi (1995). Il a repris les propos de Simmel pour montrer que "l'argent est l'instrument capable d'établir des relations très distantes dans l'espace » (Simmel cité par Giddens (Delannoi, Op.cit., p 883). Ainsi, bien qu'absent physiquement, l'individus est présent et joue pleinement son rôle auprès des siens à travers le gage symbolique. L'individu se situe dans " une organisation et réorganisation de ses relations sociales » comme l'a montré Simmel cité par Delannoi (Ibidem). Dans les rapports entre conjoints, le gage symbolique mobilisé grâce à la consommation du «garba » permet d'établir une relation de pouvoir comme l'a prouvé Halbwachs (1913). Selon l'auteur, la nourriture met en évidence les aspects cachés des relations de pouvoir et de la vie sociale, ainsi que la production de faits sociaux et de personne.

Pour réduire les risques liés à la consommation du «garba», les consommateurs ont la possibilité d'éviter l'huile des «garbadromes». Cependant, certains répondants le consomment pour des raisons de goût, tout en étant conscient du risque sanitaire. L'attitude de ces répondants vis-à-vis de l'huile des «garbadromes » en dépit du risque perçu pourrait aussi être expliqué par le fait qu'ils perçoivent ce risque comme un «risque auto imposé». Cet type de risque est plus accepté par les consommateurs que le risque technologique selon Frewer et al.(2005) et Pierrine (2019).

\section{Conclusion}

Au terme de ce travail visant à analyser l'importance du « garba » dans la construction des liens sociaux des consommateurs malgré les risques sanitaires perçus, il ressort que le «garba » est un facteur relationnel pour les individus à l'étude. En effet, les résultats ont montré que le «garba » est perçu comme un aliment de qualité sanitaire et nutritionnelle inférieure par la grande majorité des répondants. Cependant, le mode de consommation et la rationalité économique autour de l'aliment permettent aux individus d'entretenir les relations tant au niveau familial qu'au niveau extra-familial. En effet, le mode de consommation du «garba» inscrit les commensaux dans des rapports d'amitié, de fraternité. Quant à la rationalité économique autour de l'aliment, dans les rapports entre consanguins, elle permet aux ainés d'obtenir le respect 
et la considération des cadets grâce au gage symbolique que les premiers mobilisent en faveur des seconds. La mobilisation du gage symbolique par les individus issus de la classe économique modeste dans leurs rapports avec leurs géniteurs leur accorde aussi le respect et les bénédictions de ceux.

Au regard de l'enjeu social qui se structure à travers la consommation du «garba», l'on peut affirmer que le «garba» pourrait intervenir dans la construction du lien social des consommateurs. Les normes d'hygiène doivent donc aller au-delà des risques pour la santé et prendre en compte les normes socioculturelles avec une analyse participative des risques pour montrer que manger le «garba» ne conduit pas toujours à un risque pour la santé, mais apporte des avantages sociaux.

\section{Remerciements}

Cette étude a été menée dans le cadre de l'Initiative DELTAS Africa [Afrique One-ASPIRE /DEL-15-008]. Afrique One-ASPIRE est financée par un consortium de bailleurs composé de l'Académie Africaine des Sciences (AAS), l'Alliance pour l'Accélération de l'Excellence Scientifique en Afrique (AESA), l'Agence pour la planification et coordination du Nouveau Partenariat pour le Développement de l'Afrique (NEPAD), le Wellcome Trust [107753/A/15/Z] et le gouvernement britannique.

\section{References:}

1. Abouya, D. (2011). L'histoire du garba. Première Edition du festigarba à Abidjan, Abidjan, Côte d'Ivoire.

2. Anoman, T. A., Koffi, M. K., Aboua, N. K., \& Koussemon, M. (2018). Determination of ETM, Histamine and Mycotoxins in Garba, a Traditional Ivoirian Meal. American Journal of Analytical Chemistry, 9, 245-256.

3. Anoman, T. A., Koussemon, M., Kouassi, I. K., \& Ake Assi, Y. (2018). Qualité microbiologique du garba, un aliment de rue de Côte d'Ivoire. International Journal of Biological and Chemical Sciences, 12(5), 2258-2265.

4. Assanvo, B. J., Agbo, N. G., Coulin, P., Heuberger, C., \& Farah, Z. (2019). Etude comparée de 3 attiéké traditionnels et d'un attiéké commercial (Garba) : Enquêtes sur les méthodes de production et caractéristiques physicochimiques du ferment de manioc et des différents produits finis. International Journal of Innovation and Applied Studies, 26(4), 1108-1133.

5. Bardin, L. (2007). L'analyse du contenu (PUF).

6. Bassa, A. Y., Adjéhi, D., Sylvain, G. S., Koffi, M. D., \& Bassirou, B. (2016). Contamination par 
7. Bacillus cereus de l'attiéké produit dans le secteur informel au sud de la Côte d'Ivoire et gestion du risque par le réchauffage hydrothermique. International Journal of Innovation and Applied Studies, 15(3), 637-654.

8. Bourdieu, P. (1979). La distinction. Critique sociale du jugement. Paris: Minuit.

9. Corbeau, J. P. (2007). Pour une approche plurielle de notre alimentation. HOLE-DO, 104.

10. Corbeau, J.-P. (2010). La représentation et la fréquentation de la restauration rapide hamburger par les consommateurs français de 15 à 25 ans (adolescents et jeunes entrant dans la vie). Rapport commandé par l'Institut Quick.

11. Corbeau, J.-P., \& Poulain, J.-P. (2002). Penser l'alimentation. Entre imaginaire et rationalité (Ed. Privat).

12. Delannoi, G. (1995). Anthony Giddens, Les conséquences de la modernité. In: Revue française de science politique, 45ème année(5), 882-885.

13. Diabaté, M., Dossou, J., Nimaga, D., Gbogouri, A. G., \& Amani, G. N. (2018). Etude diagnostique des

pratiques de friture du thon du mets «Garba» consommé dans la ville d'Abidjan (Côte d'Ivoire).

14. International Journal of Biological and Chemical Sciences, 12(3), 1333-1344.

15. Djeni, N. T., N'Guessan, K. F., Kouamé, K. A., \& Djê, K. M. (2011). Quality of attieke (a fermented cassava product) from the three main processing zones in Côte d'Ivoire. Food Research International, 44, 410-416.

16. Dunbar, R. I. M. (2017). Breaking Bread: The Functions of Social Eating. Adaptive Human Behavior and Physiology, 3, 198-211.

17. Fischler, C. (1990). L'Homnivore (Odile Jacob).

18. Frewer, L., Fischer, A., Scholderer, J., \& Verbeke, W. (2005). Food safety and consumer behaviour. Innovation in Agri-Food Systems. Product Quality and Consumer Acceptance, 125-145.

19. Garabuau, M. I. (2002). Cuisines et indépendance (1'Harmattan).

20. Halbwachs, M. (1913). La classe ouvrière et les niveaux de vie Recherches sur la hiérarchie des besoins dans les sociétés industrielles contemporaines [Thèse de doctorat à la Faculté des lettres]. Université de Paris.

21. Hastorf, A. C. (2016). The Social Archaeology of Food Thinking about Eating from Prehistory to the Present (Cambridge University Press). 
22. Heuberger, C. (2005). Cyanide content of cassava and fermented products with focus on attiéké and attiéké garba. [A dissertation for the degree of Doctor of Natural Sciences]. ETH Zurich.

23. Koffi, A. L. J. (2013). Logiques de consommation des aliments de rue dans le district d'Abidjan: Cas du «garba» [Mémoire de DEA]. Institut d'Ethno-Sociologie, Université Félix Houphouët-Boigny.

24. Lambert, J.-L. (1987). L'évolution des modèles de consommation alimentaire en France (Lavoisier).

25. Laporte, M.-E., Michel, G., \& Rieunier, S. (2015). Mieux comprendre les comportements alimentaires grâce au concept de perception du risque nutritionnel. Recherche et Applications en Marketing(French Edition), SAGE Publications, 30(1), 81-117.

26. N'Da, P. (2006). Méthodologie de la recherche, de la problématique à la discussion des résultats, Comment réaliser un mémoire, une thèse d'un bout à l'autre (Editions Universitaires de Côte d'Ivoire, Vol. 1-3ème édition).

27. Paugam, S. (2008). Le lien social (PUF).

28. Pierrine, D. (2019). La construction de la sécurité sanitaire des aliments en milieu domestique en France (Maine-et-Loire). Socioanthropologie, 39, 25-38.

29. Poulain, J. P. (2002). Manger aujourd'hui: Normes, attitudes, pratiques (Editions Privat).

30. Poulain, J.-P. (1998). Les jeunes seniors et leur alimentation. Cahiers de l'OCHA, 9.

31. Tibère, L. (2006). MANGER CREOLE: Interactions identitaires et insularité à La Réunion. Ethnologie française, Vol. 36(3), 509-517.

32. Vaughan, J. P., Morrow, R. H., \& Organization, W. H. (1991). Manuel d'épidémiologie pour la gestion de la santé au niveau du district. 